\title{
On the Positive Role of College Vocal Music Teaching in the Protection of Intangible Cultural Heritage
}

\author{
Chenglong Cui \\ College of Arts, Yanbian University, Yanji Jilin, 133002, China
}

Keywords: Colleges and universities, Vocal music, teaching, Intangible cultural heritage, Protection.

\begin{abstract}
The vocal music teaching in colleges and universities has very unique advantages and significance in the protection of intangible cultural heritage. Through the combination of the above two, we can better inherit the excellent traditional culture of the Chinese nation, help the society develop more urgently needed talents, promote the characteristics of higher education institutions, and help more teachers achieve rapid growth. This paper expounds the urgency of integrating intangible cultural heritage protection into vocal music teaching in colleges and universities, analyzes the positive role of implementing intangible cultural heritage protection in college vocal music teaching, and puts forward the further development of non-material cultural heritage in college vocal music teaching, and the protection of the active role of heritage protection.
\end{abstract}

\section{Introduction}

Under the current trend of world economic integration, people are enjoying greater and greater negative pressures while enjoying the great benefits of economic and social development. For example, the problem of homogenization of culture due to the process of globalization has greatly affected our national cultural traditions and cultural heritage. In order to protect all types of intangible cultural heritage to the greatest extent possible, UNESCO has already introduced the Convention on the Protection of Intangible Cultural Heritage. The development of vocal music education in China's colleges and universities has been implemented for more than 100 years. Although great achievements have been made, there are still many deficiencies, including the protection of the intangible cultural heritage of music. Lack of education is the most significant deficiencies. Therefore, it is of practical significance to integrate the protection of intangible cultural heritage into the teaching of college vocal music. It is also worth paying close attention to every college vocal music education worker.

\section{The Urgency of Integrating Intangible Cultural Heritage Protection into Vocal Music Teaching in Colleges and Universities}

On the one hand, it is necessary to carry out the protection of intangible cultural heritage. The reason why the protection of intangible cultural heritage has received so much attention and attention is that the protection of intangible heritage is of great significance. The protection of intangible cultural heritage is the crystallization of public wisdom for thousands of years, and it is also an important spiritual pillar that supports the Chinese nation in standing among the nations of the world. In the new situation where the world's cultural economy and integration tend to be integrated, strengthening intangible protection is an important means of ensuring the independence of the Chinese nation's culture. As China enters a period of rapid development, the people's lifestyle, environment, and spiritual pursuits have all undergone tremendous changes. The environment in which non-material cultural heritage protection depends on survival has been destroyed, resulting in the development of non-heritage projects. Difficult, even a large number of intangible items have disappeared. The state has issued many laws and regulations regarding the protection of intangible cultural heritage, and has promoted nationwide non-survival census work, creation of an intangible 
list work system, selection of non-genetic inheritors, creation of cultural reserves, creation of non-genetic institutions or The contents of the exhibition center, etc., allow the intangible cultural heritage to be well protected. However, due to the complexity of the incommunicado, the creation of laws and regulations is difficult to completely harmonize with the protection of non-heritage. The attention to the protection of intangible cultural heritage across the country is inconsistent, and the collection, sorting, display, and personnel training related to protection are inconsistent. Such issues are relatively weak, and there are a large number of problems owed to funds and staff. A large number of intangible projects lack inheritors and face the important issue of the disappearance of inherited projects. Intangible protection and inheritance cannot be dominated by the government alone. It is also urgent for universities and other social forces to participate.

On the other hand, it is the need for the sound development of college vocal music teaching. As early as in 2008, the Ministry of Culture announced the main points of China's intangible protection work and requested that intangible education be included in the national education system so that young people can reflect the charm of intangibles more. Inheritance into colleges and universities has also put forward higher requirements for teaching, and fewer teachers have strong inheritance. All kinds of colleges and universities are building comprehensive courses. University vocal music teaching uses a large number of teaching methods in music schools. The content of teaching involves very little local intangible content. It is very inappropriate for a practical new teaching model. To increase the content of intangible cultural heritage in college vocal music teaching can effectively meet the needs of society and is also a requirement for the development of higher education in China. For college vocal music teachers, the related work of increasing the protection of intangible will be expanded, the contents of education and teaching will be expanded, relevant courses will be set up, the related papers will be drafted, the topics of non-heritage areas will be declared, and books and vocal works will be published. Conduct innovative research. Universities around the country should pay attention to the social needs and the individual development of vocal music teachers in the cultivation of the vocal talents for the projects. All of them will have very great needs for intangible protection.

\section{The Positive Role of Implementing Non-material Cultural Heritage Protection in Vocal Music Teaching in Colleges and Universities}

The protection of intangible cultural heritage is a very broad concept and can be mainly characterized by liveliness, inheritance, and rheology. This will be discussed below.

First, vocal music education in colleges and universities can train inheritors to play a protective role in non-living activities. Non-heritage projects that have a direct bearing on college vocal music teaching are mainly reflected in traditional folk songs, traditional folk arts, and traditional operas in traditional performing arts venues. The most closely related ones are folk songs. The legacy of performing arts in the intangible cultural heritage has a lively character. This feature is the core content of understanding the protection of performance cultural heritage. The cultural meaning of the protection of intangible cultural heritage is demonstrated by human activities, and the vitality of non-living cultural heritage can precisely demonstrate the important value of inheritors. In college vocal music teaching, non-genetic inheritors can be invited into the classroom for teaching, so that college students actively learn from non-genetic inheritance. At the same time, colleges and universities can also use vocal music teaching to cultivate new inheritors of intangible projects. At present, the Chinese government has financial support for the inheritors at the national and provincial levels, while the private sector is also facing the problem of non-inheritable reductions. Therefore, non-genetic inheritance training should be properly integrated into college vocal music education, and vocal music teachers can often master the relevant technologies of intangible projects through learning, thus becoming a veritable inheritor.

Second, academic research on vocal music teaching plays a protective role in non-genetic inheritance. The inheritance of intangible cultural heritage mainly means that it possesses the characteristics of human collective or individual inheritance and development from generation to 
generation. Intangible cultural heritage is mainly transmitted in the form of oral and mental teaching. It is very important to be able to record and preserve the inheritor's skills in a timely and accurate manner. The academic research in vocal music education can carry out field investigations on the status quo of the intangible cultural heritage protection, recording, recording and recording vocabulary, and creating a database of inheritors' archives. It can be confirmed by using documentary records, material carriers, and verbal materials. This infers the original appearance of the intangible cultural heritage at a particular period, thus combing out its course of development, and inheriting the skills of the inheritor to better inherit all kinds of intangible cultural heritage. Colleges and universities should carry out in-depth academic research on intangible cultural heritage in the process of vocal music teaching, so as to provide corresponding theoretical system support for the next step in the protection of intangible cultural heritage.

Third, college vocal music teaching practice plays a protective role in non-residue rheology. The protection of intangible cultural heritage will change as the times change. In order to effectively meet the actual needs of modern people, different forms emerge under different social and historical conditions. This is actually the rheological characteristic of intangible cultural heritage. For example, the state-level intangible cultural heritage protects Fengyang Huagu, which is usually performed by two people with a dozen gongs and drums before the liberation. After the liberation, in order to be able to appreciate it better, the Fengyang hubbub has become an art form that takes many people's dance as its main form while singing and dancing. This is very different from the art form that focuses on singing. In the process of external communication of intangible cultural heritage, it is often fused with local language and culture to form a new art form. By understanding the rheological characteristics of the intangible cultural heritage, we have made it clear that the intangible cultural heritage should be protected, recorded, and passed on.

\section{Measures to Further Play the Positive Role of Intangible Cultural Heritage Protection in College Vocal Music Teaching}

The first is to carry out work in light of local conditions. Some researchers believe that the protection of non-heritage should protect the so-called cultural space in which it survives and develops. However, if you want to preserve the space authentically, there is a great deal of difficulty. Under the circumstances of world economic integration, the natural and social environment in which Chinese traditional culture depends on and survives has undergone tremendous changes. A large number of intangible projects have lost the soil on which they rely, and blindly recovering is obviously not in line with reality. . If it is necessary to genuinely protect the cultural space of non-heritage, then there is only one way of thinking, that is, letting history stay at a certain point in time, which naturally cannot be achieved. At the same time, we should also be clearly aware that the essence of inheritance and protection of intangibles is to under the guidance of the concept of lively protection and respect for new ideas, so that the non-evil sense and its core values can be better served The construction of a harmonious society in our country. In view of this, the vocal music teaching in institutions of higher learning should be combined with the scientific implementation of the protection of intangible cultural heritage. We must try our best to adapt our measures to local conditions and measures, and we should not be too full of blame. We must not be afraid because we have been unable to restore the original environment. Otherwise, we will become stiff and rigid. The realistic cultural environment where insurgents can survive should be simulated as much as possible within the limits of their capabilities, and efforts must be made to ensure that the core elements are not lost and that the integrity of the form can be basically guaranteed. In fact, even on the stage of the CCTV young singer grand prix, those players of the original ecological group have also used modern music equipment such as microphones and stereos. But who said that what they displayed is not an insignificance?

The second is to focus on field gatherings. In ancient China, folk songs were called wind, and folk songs were collected to call it the wind. Today, Erquan Yingyue is already a well-known Chinese traditional music, and it all relied on Yang Yinliu, the founder of the People's Republic of China, to 
try to find some artists who were facing extinction in order to rescue some of the extinct folk music wealth. Joining the collection style without Mr. Yang, people nowadays can hardly hear the erhu title that the first person touched, so the work of collecting wind is very important. Colleges and universities generally give students a certain time to go out to collect fields, but some teachers and students are completely in the form of external, and even consider this learning content as outings. This is a pity. Therefore, it is necessary to pay full attention to the field work of collecting wind, which is also an important source of implementing the protection of intangible cultural heritage. It is necessary to carefully select destinations for collecting winds and to comprehensively understand the ethnic conditions, basic history, cultural environment, and artistic forms in this area in advance, and produce basic outlines for collecting winds. At the same time, we should fully use the convenient conditions under the conditions of modern science and technology, and use recording equipment, cameras, cameras, etc., to fully record the progress of wind collection and drill open. On this basis, not only should the intangible project itself make a specific record, but it should also record information relating to its culture, history, and dissemination. It should also promptly report on the report of the collection of the wind after the end of the harvest.

The third is to actively cultivate representative inheritors. The most important carrier for non-heritage is human beings. The death and death of arts and people walking along with people is a full expression of the fragility of the protection of intangible cultural heritage. In 2006, China had formulated and implemented the Interim Measures for the Identification of Representative Inheritors of State-level Intangible Projects, and gradually named and announced the inheritors of state-level non-heritage projects. Each region is also in the process of implementing provincial-level non-inherited project successors. By observing this list, it can be found that few of the inheritors come from schools, and that they are generally very old. Colleges and universities are an important place for inheriting modern human civilization. Can we cultivate non-genetic inheritors among university teachers and students, and the exploration in the field of vocal music teaching in colleges and universities seems to be of significance.

\section{Summary}

In general, colleges and universities are important places for protecting, inheriting, and developing civilizations. College vocal music teaching has a very obvious advantage in protecting intangible cultural heritage. As long as a scientific university is planned and used actively and reasonably, the teaching of college vocal music courses will definitely make new and greater contributions to the protection of intangible cultural heritage in China. The key to this is to continuously strengthen the development of related materials and focus on field work. , and implement relevant work according to local conditions. The author firmly believes that with the active play of college vocal music teaching, the protection of intangible cultural heritage in China will inevitably make greater progress.

\section{Acknowledgement}

This research was financially supported by the Protection and Inheritance of "Yanbian Korean Intangible Cultural Heritage" by National Vocal Music Teaching.

\section{References}

[1] Song Junxiong, Wang Kaitao. Non-material cultural heritage protection research, Guangzhou: Sun Yat-sen University Press, 2013.

[2] Liu Chenghua. A Study on the Particularity of Protecting the Cultural Heritage of Intangible Cultural Heritage, Nanjing: Nanjing University Press, 2015.

[3] Liao Mingjun, Yang Minkang. Traditional Music and Intangible Cultural Heritage Protection, National Art, 2008(1). 
[4] Dai Hong. The Inheritor of Traditional Music in the Perspective of Non-material Cultural Heritage Protection, Journal of Xinghai Conservatory of Music, 2009(1).

[5] Zhou Heping. The practice and exploration of China's intangible cultural heritage protection, Qiushi Journal, 2010(2). 